\title{
CLASSIFICATION OF PARAMETRIC DESIGN TECHNIQUES - TYPES OF SURFACE PATTERNS
}

\author{
${ }^{1}$ Réka SÁRKÖZI, ${ }^{2}$ Péter IVÁNYI, ${ }^{3}$ Attila Béla SZÉLL \\ ${ }^{1}$ Breuer Marcell Doctoral School, Faculty of Engineering and Information Technology, \\ University of Pécs, Boszorkány u. 2, H-7624 Pécs, Hungary, \\ e-mail: sarkozi.reka.00@mik.pte.hu, \\ ${ }^{2}$ Department of System and Software Technology, Faculty of Engineering and Information \\ Technology, University of Pécs, Boszorkány u. 2, H-7624 Pécs, Hungary, \\ e-mail: ivanyi.peter@mik.pte.hu \\ ${ }^{3}$ Department of Building Construction, Faculty of Engineering and Information Technology, \\ University of Pécs, Boszorkány u. 2, H-7624 Pécs, Hungary, e-mail: szellattila@mik.pte.hu
}

Received 1 January 2016; accepted 12 April 2017

\begin{abstract}
The main objective of this paper is to classify the techniques of parametric design, and to make the existing classification system more exact. The previously created classification was based on shape and logical aspects, which granted a good approach for parametric design techniques and helped providing an overview. However this system, for the sake of scientific precision, needed further darification at points. In this paper a more precise classification is shown, which is based on the interpretation of patterns as graphs. This enables their topology to be studied in a more exact, mathematical way.
\end{abstract}

Keywords: Generative design, Parametric design, Structural design, Structural shapes

\section{Introduction}

In order to understand and learn how to use parametric design to shape a building it is very useful to understand the possible and usable forms and patterns. These parametric structures are generated by commands of the design program, which is very similar to a programming language. This results in the design of structures, which are based on geometry and mathematics. Using a classification system that is based on the mathematical properties of these structures it is easier to understand how they were made and easier to learn, how other similar structures can be designed and how many possibilities are available. There is also a need in this new field of architecture to explore and describe the generative process of design, also called as Parametric Design Thinking (PDT) [1]. This paper is a part of a possible answer to this and the question, 
whether the pattern of a structure is a tiling or a subdivision is one of the first questions when classifying a structure in this way.

In a previous research [2] the classification was based on shape and logical aspects, where mathematically similar structures made up a class. It was based on many different sources [3]-[8] and as a result of these, the geometric properties provided the most basic selection rules. The previously published method is similar to the present classification system, but it requires further refinement. This paper shows how the refinement of the surfaces group of the previous classification system is represented.

In the previous paper [2] two elementary categories of parametric design techniques were differentiated: surfaces and formations. Surfaces were defined as structures, which consist of a pattern on a planar or curved surface. Formations were defined as structures that occupy a more extensive part of space. Since a clear borderline between the groups is absent, the deciding rule is that if the parametric structure has a structural role then it is a formation. Modifiers were mentioned as a third group, which modify the members of the previous two groups. This paper focuses solely on the surfaces as other parts of the group that need further clarification.

The sources used to this paper also deal with the part of the parametric design, which cover the tiling-subdivision-packing theme, because of its importance. In the work of Jane and Mark Burry [3] packing and tiling are mentioned and in the book of Jabi [4] both tiling, packing and subdivision are distinguished, where packing is defined as "the placement of many objects in space, in a way that little or nothing of it is left over" and subdivision is referred as a division of surfaces and generation of meshes in a way, that the results are suitable for Computer Numerical Control (CNC) machines.

\section{Classification of surfaces}

The two properties of surfaces described in [2] are also used here, namely form and pattern. The classification of the form of surfaces has a full mathematical foundation, and a surface can be parabolic, elliptic, hyperbolic and complex in form. However the definitions of the grouping of the types of patterns should be changed as follows.

- Patterns remaining on the surface:

○ tiling;

○ subdivision;

$\circ$ packing - the content of this group didn't change, but it will be called lacunary pattern in future for the sake of clarity;

- Tridimensional patterns:

○ open;

- closed.

The classification remained unchanged from the aspect that tridimensional patterns are considered as a three dimensional extension in the aspect of patterns remaining on the surface. The significant changes concern tiling, subdivision and lacunary patterns. Two pattern types, tiling and subdivision will be the principal topologies while in the future the lacunary pattern will be a modification of these two. 
The main difference between tiling and subdivision is that tiling focuses on making identical or analogue tiles while subdivision means dividing the surface at random tiles by applying some rules. In other words the purpose of the first one is the creation of tiles, while the second is the division of the surface, as it is indicated by the name. To accurately define the difference mathematically it is needed to interpret these patterns as graphs. Using graphs to construe difficult models and patterns of the real world is a good working method, which can be seen in a wide range of scientific research [9]-[13].

Another modification can be made in the classification, that the mesh, which will be mentioned as the foundation of making the pattern instead of a type of subdivision as in paper [2], because the pattern is created by a mesh in every instance. This is a transition between the physical appearance of the pattern and the full theoretical graph-like interpretation.

\section{Topology of patterns}

The patterns are interpreted as undirected simple planar graphs. This means that edges have no orientation, both multiple edges and loops are disallowed and vertices and edges can be drawn in a plane as long as no edge intersects with any other edge.

This means that subdivision is a pattern, for which - interpreted as a graph - one of the following is true:

- the number of neighbors inside vertices varies; or

- the number of vertices and edges of inner cycles varies,

where a cycle is a continuous series of vertices and edges, where each member participates only once and the point of origin is identical to the point of arrival.

Tiling is a pattern, for which - interpreted as a graph - the following conditions are both true:

- the number of neighbors of inside vertices are equal; and

- the number of vertices and edges of inner cycles are equal.

The side vertices and edges of the graphs do not obey this law.

A pattern is called lacunary pattern, when the interpretation of the graph can be tiling or subdivision; however the surface contains uncovered areas.

Two examples are presented for this thesis, which provide a better contrast between the groups.

\section{Example 1}

\subsection{Subdivision}

The main idea of the Voronoi-diagram is that the surface is divided into cells based on a predefined set of points, where every point of the surface belongs to the cell of that predefined point to which it is closest. In this example the set of points is taken at random, therefore the cells will be irregular. It is possible for a vertex to have more than 
three edges, for example when the points of the set, which were the base points of the Voronoi-diagram, are the points of a square mesh, the Voronoi-diagram is also a square mesh, and each of its vertices have four edges. However, when the set of points are truly random, three edges in a vertex are guaranteed, as it is demonstrated in Fig. 1c. Though some vertices can be seen as it has four edges at first glance, there are actually two points connected by a tiny edge, which cannot be seen at this magnification. However the cells, which create the cycles in the graph, are different polygons, which consist of a variable amount of vertices and edges. So the Voronoi-diagram - when it is made of random points - can be considered as a subdivision.

An interesting phenomenon is that when the points of the set, which were the base points of the Voronoi-diagram, are connected with their neighbors then it forms a triangle mesh. Those points are considered neighbor points when Voronoi-cells have a common edge. On a graph of this mesh the cycles always contain three vertices; however the number of neighboring vertices of a vertex is variable. This is the dual of the Voronoi subdivision and it is called the Delaunay triangulation, as it is shown in Fig. $1 b$.

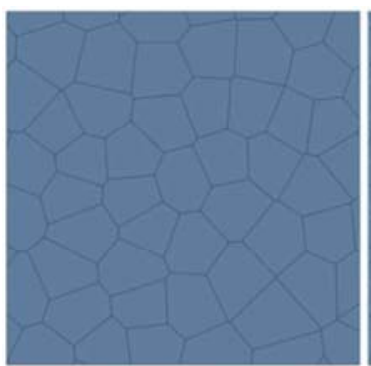

a)

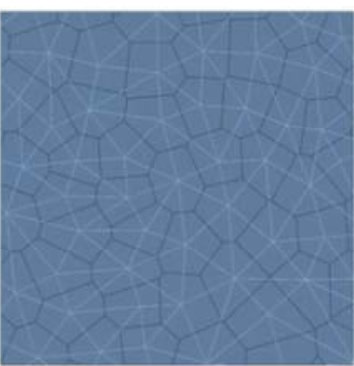

b)

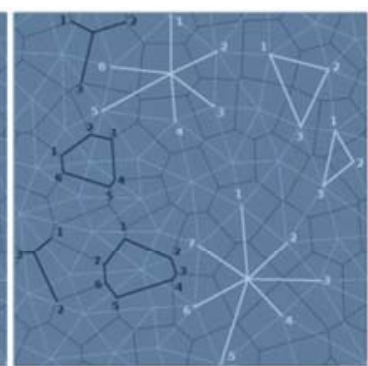

c)

Fig. 1. a) Voronoi-diagram; b) Voronoi-diagram with the mesh made from its initial points; c) Number of neighbors of vertices and number of vertices of cycles in the Voronoi-diagram and in the mesh

\subsection{Tiling}

In the case of hexagonal tiling the Voronoi division is also applied through the initial points of the cells are set to provide regular hexagonal tiling. If a plane is to be divided into regular hexagons with Voronoi division, then the vertices have to be taken of a regular triangular mesh as the initial points of cells. The cells created in this way are all regular hexagons, then the initial points of cells have to be the vertices of a regular triangular mesh, so in the case of the graph interpretation of the pattern the inside vertices belong to the same amount of edges and the inside cycles consist of the same number of edges and vertices, as shown in Fig. 2. Therefore this method is considered as tiling.

In the case when a similar pattern is applied on a hyperbolic - or any non-planar surface, then the hexagons are projected to the surface, but the topology remains 
unchanged. As it shown in Fig. $2 d$, the hexagons are not perfectly equal, but it still remains as tiling.

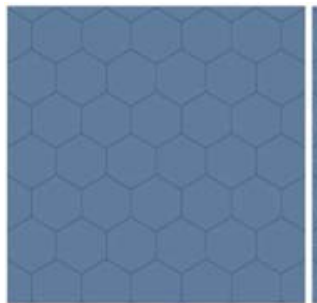

a)

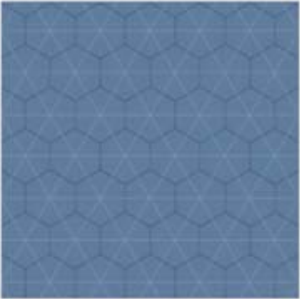

b)

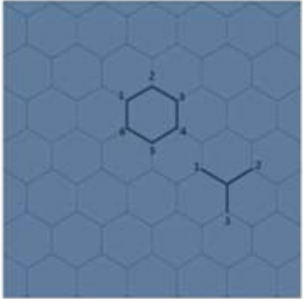

c)

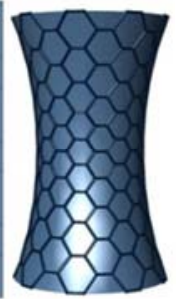

d)

Fig. 2. a) Hexagonal tiling; b) Hexagonal tiling with the regular triangular mesh made from its initial points; c) Number of neighbors of vertices and number of vertices of cycles in the hexagonal tiling; d) Hexagonal tiling on hyperbolic surface

\subsection{Lacunary pattern}

The third example is circle packing. This is created by taking the triangular mesh used at the Voronoi division then this mesh is optimized so that tangent circles can be created [14]. This optimization does not concern the topology of the mesh; it merely changes the position of points and the length of the edges. So far the mesh, which helped to create the circles makes the statement that the inside cycles have the same number of edges and vertices (three) true. But not the same number of edges belong to the inside vertices, as it is shown in Fig. 3e. So this lacunary pattern is derived from subdivision.

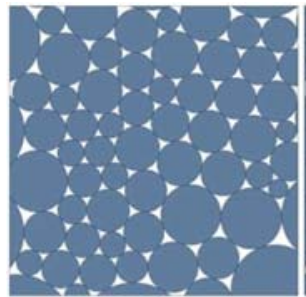

a)

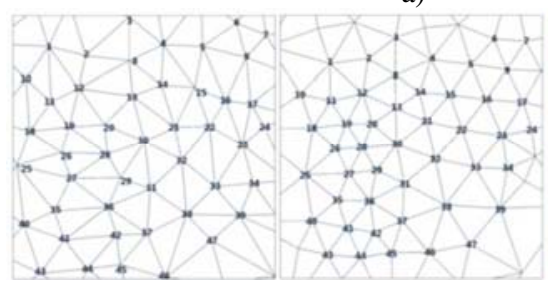

c) d)

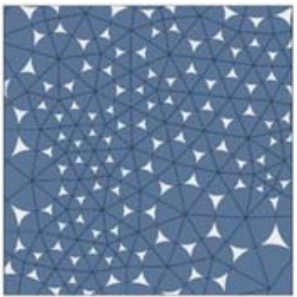

b)

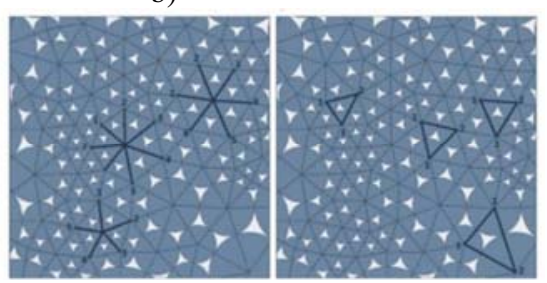

e)

Fig. 3. a) Circle packing; b) Circle packing with the mesh made from its initial points; c) Mesh of the original Voronoi-diagram; d) Optimized mesh used to circle packing; e) Number of neighbors of vertices in the mesh of the circle packing; f) Number of vertices of cycles in the mesh of circle packing 
The original name 'packing' comes from 'circle packing' [14], which means that most circles try to squeeze (pack) into a rectangle 'box'. That is why the 'packing' term is a good match with reality in this case, but as the second example shows it is not suitable in every instance. The term 'packing' in general is used for geometric problems, when a rectangle or a box is be to filled in the densest way with certain planar shapes or bodies. That is why we changed the name of the group to a lacunary pattern.

In every case the base of these patterns was the Voronoi-diagram or its initial points. Patterns are created by dividing rectangles in the second example.

\section{Example 2}

\subsection{Subdivision}

In this case the starting point contains five identical rectangles, which are divided into two with a vertical line. The position of these lines is random; therefore the five rectangles are divided at different positions. The resulting ten different rectangles are divided into two again, horizontally this time and again at random. Then those from the newest rectangles whose area exceeds a certain size are divided again vertically, similarly to the previous divisions. The result is a surface consisting of rectangles of different areas and ratios, which appears as a surface covered with totally random rectangles. It can be seen from the transition of the pattern to a graph that every inner vertex has three neighbors, but a cycle can consist of four, five, six, even eight vertices. This subdivision is shown in Fig. 4. Theoretically it is possible for the vertices to have four neighbors, in common with the Voronoi division, if the neighboring rectangles are divided at the same place similar to the Voronoi division.

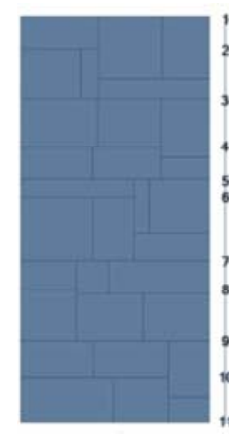

a)

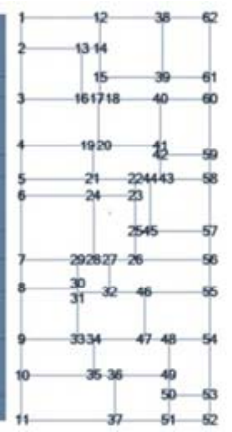

b)

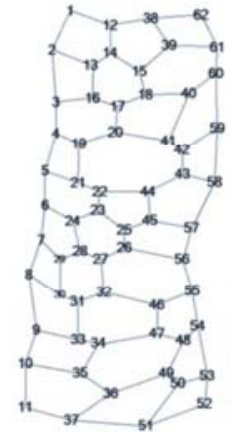

c)

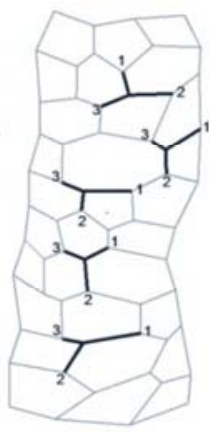

d)

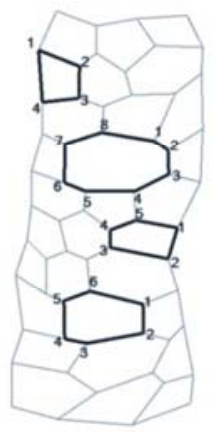

e)

Fig. 4. a) Subdivision of rectangles; b) Numeration of vertices of rectangles;

c) Tiling converted to a graph with the same numeration; d) Number of neighbors of vertices in the graph; e) Number of vertices of cycles in the graph 


\subsection{Tiling}

If the base rectangles are divided with the previous set of rules, but always at the half of the edges, the result will be a pattern consisting of identical rectangles, where every vertex has four neighbors and every cycle has four vertices, as it is shown in Fig. $5 a$ and Fig. 5b. If this pattern is applied on a non-planar surface, the rectangles may not be identical but the topology will not change, as in the case of the hexagonal tiling.

\subsection{Lacunary pattern}

In the case of a lacunary pattern the tiling is modified by pulling certain vertices apart, which result in rhombuses. In this case it can be seen in Fig. $5 c$ and Fig. $5 d$, that every cycle consists of four vertices but one vertex has three or six neighbors. However it is a tiling as well if there are different types of vertices and cycles, but this difference comes from the decision of the designer and is not due to randomness. Because these types of vertices are predefined it is also tiling made up of two tiles. When one type of tile is not used, it results in a lacunary pattern. Most historical architectural patterns are tilings, which consist of more than one type of tile [15], [16].

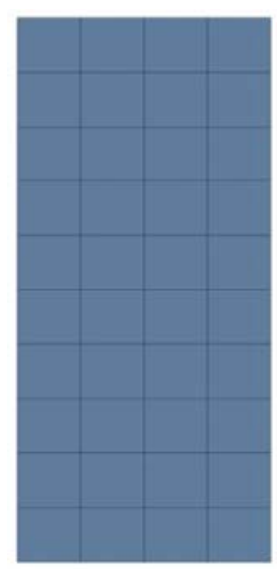

a)

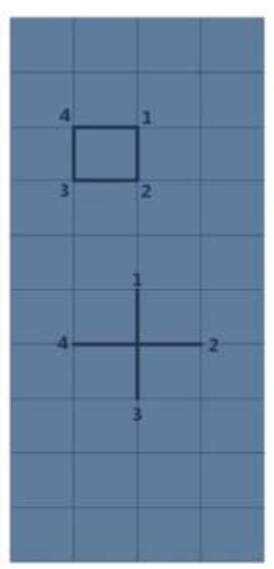

b)

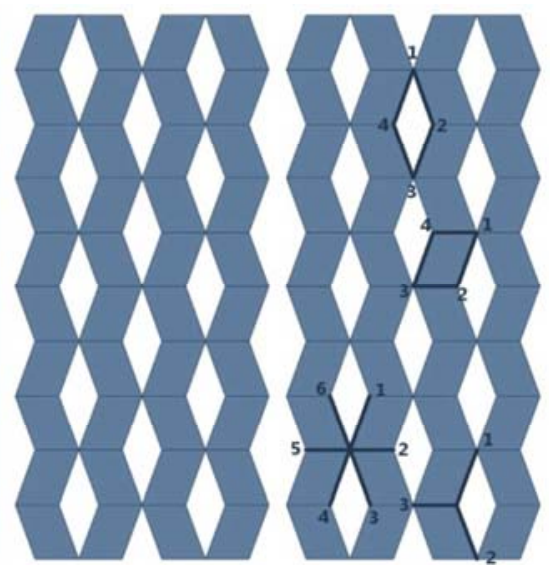

c)

d)

Fig. 5. a) Rectangle tiling; b) Number of neighbors of vertices and number of vertices of cycles of the tiling; c) Lacunary pattern made from rectangle tiling; d) Number of neighbors of vertices and number of vertices of cycles of the lacunary pattern

\section{Conclusion}

By representing the patterns as graphs, it helps to construct a clear and simple to use topological classification for patterns of parametric design techniques. Because the surface modifying patterns are derived from the patterns remaining on the surfaces their topology is identical. A final question that may come up is whether this classification could be extended to formations as well. This question is subject to further research 
because while some formations have a clear, easy to see topology, others need to be further reflected upon.

\section{References}

[1] Oxman R., Gu N. Theories and models of parametric design thinking, Proceedings of the 33rd International Conference on Education and Research in Computer Aided Architectural Design in Europe, Vienna, Austria, 16-18 September, 2015, pp. 477-482.

[2] Sárközi R., Iványi P., Széll A. B. Classification of parametric design techniques, Proc. of the Fifteenth International Conference on Civil, Structural and Environmental Engineering Computing, Prague, Czech Republic, 1-4 September 2015, paper 227.

[3] Burry J., Burry M. The new mathematics of architecture, Thames and Hudson Ltd, NY, 2012.

[4] Jabi W. Parametric design for architecture, Laurence King, London, 2013.

[5] Krish S. A practical generative design method, Computer-Aided Design, Vol. 43, No. 1, 2011, pp. 88-100.

[6] Tadeschi A., AAD: Algorithms Aided Design, Le Penseur Publisher, Brienza, 2014.

[7] van Wijk J. J., Cohen A. M. Visualization of Seifert surfaces, IEEE Trans. on Visualization and Computer Graphics, Vol. 12, 2006, pp. 485-496.

[8] Catmull E., Clark J. Recursively generated B-spline surfaces on arbitrary topological meshes, Computer-Aided Design, Vol. 10, No. 6, 1978, pp. 350-355.

[9] Cardillo A., Scellato S., Latora V., Porta S. Structural properties of planar graphs of urban street patterns, Physical Review E, Vol. 73, No. 6, 2006, pp. 1-18.

[10] Yeh Y-T., Breeden K., Yang L., Fisher M., Hanrahan P. Synthesis of tiled patterns using factor graphs, ACM Transactions on Graphics, Vol. 32, No. 1, Article 3, 2013, pp. 1-13.

[11] Foltête J. Ch., Clauzel C., Vuidel G. A software tool dedicated to the modeling of landscape networks, Environmental Modeling \& Software, Vol. 38, 2012, pp. 316-327.

[12] Erös T., Olden J. D., Schick R. S., Schmera D., Fortin M-J. Characterizing connectivity relationships in freshwaters using patch-based graphs, Landscape Ecology, 2012, Vol. 27, No. 2, 2012, pp. 303-317.

[13] Jablan S. Knots and links in architecture, Pollack Periodica, Vol. 7, No. Supplement, 2012, pp. $65-76$.

[14] Höbinger M. Packing circles and spheres on surfaces, Diploma Thesis, Institute of Discrete Mathematics and Geometry, Vienna University of Technology, 2009.

[15] Dabbour L. M. Geometric proportions: The underlying structure of design process for Islamic geometric patterns, Frontiers of Architectural Research, Vol. 1, No. 4, 2012, pp. 380-391.

[16] Bérczi Sz. Geometry of royal tomb art of ancient Eurasia: Scythian, Xiongnu and Chinese Ethnomathematics from Ukraine, Russia, Mongolia and China, Pollack Periodica, Vol. 7, No. Supplement, 2012, pp. 199-211. 